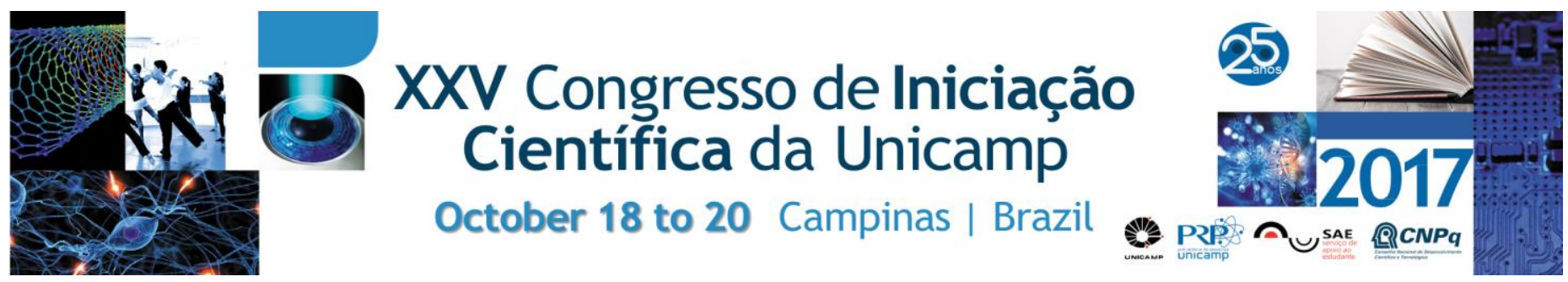

\title{
Simulator of Medical Emergency for Training - Interactive Interface Module
}

\section{Otávio V. Miranda*, Leonardo C. Villani Filho, Francisco Nardi.}

\begin{abstract}
This project involves the development of a web application intended to produce a virtual training system for students of medicine. The software is designed to generate realistic medical scenarios in a friendly, engaging manner, fostering the practice of their knowledge even in the earlier stages of their courses. This research focuses on the interface aspects.
\end{abstract}

\section{Key words:}

Medical, emergency, simulation, training, interactive, software, realistic scenarios, practice.

\section{Introduction}

This research project encompasses the conception and development of a medical emergency simulation game intended for teaching activities. It has medicine undergraduate students as its target audience.

The system will be the basis of a practice and improvement platform for medical students. It allows undergraduate students to test their knowledge in an environment free of the pressures of a real hospital emergency room. This proposal innovates due to its architecture built on top of a knowledge base, allied to an interactive simulation interface.

In the current stage, both the database access and the dynamic interface modules have been implemented. The first module makes queries to the database and prepares the received data to be utilized by the interface. The second module adapts the received data for specific interfaces to enable interactivity with the user. The result is a system that allows the generation of a dynamic interface based on the server's data.

\section{Results and Discussion}

In the current stage of development, the dynamic interface can interpret the data received from the server and display it to the user.

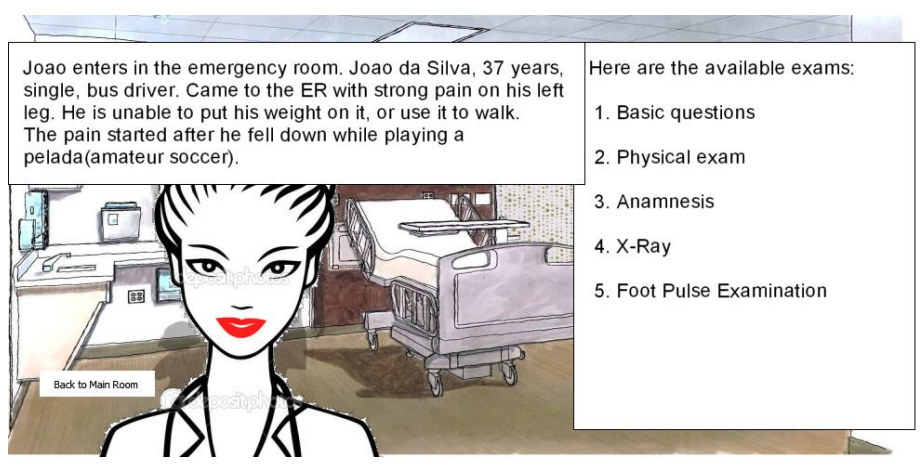

The menu with exam or treatment options is filled on demand with data that describes each clinical case, allowing variations according to the case.

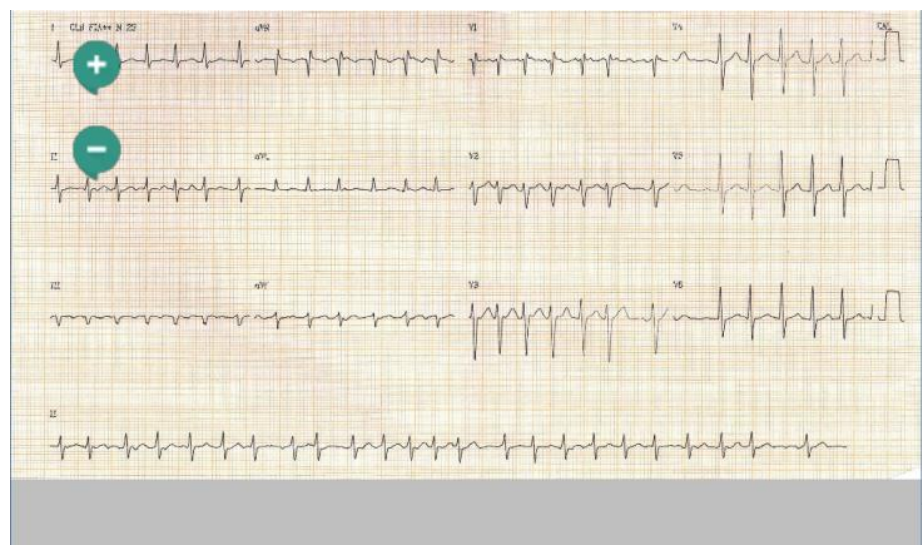

If necessary, realistic exam results may be displayed to the player for analysis, enabling the description of complex cases.

\section{Conclusions}

In the present stage of the project, many of the most important features have been implemented. Future work involves further refinement and appropriate art assets as well as tests with the students.

\section{Acknowledgement}

Work partially financed by CNPq PIBIC (800295/2016-1), FAPESP/Cepid (2013/08293-7), INCT in Web Science (CNPq 557128/2009-9), and individual grants from CAPES and CNPq. 